\title{
On the Mechanism of the Step Coverage of Blanket Tungsten Chemical Vapor Deposition
}

\author{
J. E. J. Schmitz \\ Genus, Inc., Thin Film Division, Sunnyvale, California 94089 \\ A. Hasper ${ }^{b}$ \\ MESA Research Institute, University of Twente, P. O. Box 217, 7500 AE Enschede, The Netherlands
}

\begin{abstract}
In this study, computer modeling of the contact fill process with chemical vapor deposition, (CVD) of tungsten is used to show the importance of several details on the quality of the fill process. The effect of surface curvature on the step coverage of CVD-W has been investigated. It is shown that for contacts with an aspect ratio smaller than one, the effect of surface curvature is substantial and actually improves step coverage. Therefore, surface curvature for features with aspect ratios smaller than one, should be accounted for in computer simulations of the fill process. For contacts with aspect ratios larger than one the effect of surface curvature is negligible. It is shown that the size of the void (which will be formed in cases of step coverage less than $100 \%$ ) is a better way to describe the quality of the deposition and the repercussions of the void on subsequent process steps such as tungsten etch back. In addition, the size of the void depends for a given set of deposition conditions solely on the depth of the contact rather than the contact diameter (for aspect ratios larger than 1.0).
\end{abstract}

Blanket chemical vapor deposition of tungsten (CVD-W) deposited under optimum conditions is known to be capable of voidless filling of high aspect ratio contact and via windows in sub-micron (very large scale integrated/ultra large scale integrated) (VLSI/ULSI) circuits. This technique of planarization of contacts is not only extremely important from a reliability point of view but it also opens several avenues for optimized design rules for the layout of multi-level interconnect systems such as stacked vias and non-capped contacts. ${ }^{1}$

A prime factor determining the success of a planarization step involving CVD is the problem of void formation. See Fig. 1 for an illustration of this phenomenon. The presence of a void in the planarized feature can lead to killing defects in subsequent etch back steps. It is generally believed that the higher the aspect ratio of the contact the more difficult it is to fill (i.e., void formation can easily occur). This idea is also supported by analytical solutions of diffusional equations (see below) describing mass transport into pores. In this paper, however, we will show that to a first approximation (as it is implemented in analytical solutions) only the depth of the contact is important in determing if void formation will occur for a given set of conditions. With a more refined approach we will also show that the initial aspect ratio (i.e., not only the depth but also the radius) of the contact can play a role as well, but for a reason which has not been identified before in the literature. In addition, some comments on the usefulness of the concept of step coverage will be made.

\section{Theory}

A parameter which describes the conformality of a set of deposition conditions is the step coverage (see Fig. 2a for a definition of step coverage in this study). We define $100 \%$ step coverage as being the capability of a deposition process to exhibit identical deposition rates on every surface exposed to the gas phase. If a $100 \%$ step coverage condition during the entire hole fill process is present, voidless filling of contacts will occur. In order to achieve this, sufficient transport of reactants into the contact and products out of the contact is necessary, such that depletion (of reactants) or accumulation (of products) is prevented. In addition, the reactor should not run in a reactant starved mode (gradientless reactor). In other words, the rate of the deposition is solely controlled by surface concentrations of the reactants.

\footnotetext{
Present address: SGS-THOMSON, 38921 Crolles Cedex, France.

'Present address: TEMPRESS B.V., 7900 Hoogeveen, The Netherlands.
}

From this it is clear that mass transport plays a key role in the description of step coverage. In principle several mass transport mechanisms can be present, for example surface diffusion (2-D gas) and gas phase diffusion (Fick's). It has been found, however, that in the case of CVD-W the deposition rate in high aspect ratio contacts can accurately be described by gas phase diffusion and chemical reaction rate theory only and that other mechanisms like surface diffusion probably play a minor role. ${ }^{2.3}$ Fortunately, the chemical reaction rate of CVD-W, based on the $\mathrm{H}_{2}$ reduction of $\mathrm{WF}_{6}$, has been well studied and is found to follow $\mathrm{w}^{4.5}$

$$
\text { Deposition rate }=k \times\left[\mathrm{P}_{\mathrm{WF} 6}\right]^{0} \times\left[\mathrm{P}_{\mathrm{H} 2}\right]^{1 / 2}
$$

where $k$ is the reaction constant at a given temperature, and $\mathrm{P}_{\mathrm{H} 2}$ and $\mathrm{P}_{\mathrm{WF} 6}$ are the partial pressures of hydrogen and tungstenhexafluoride, respectively. This rate equation and the mass transport equations allow one to simulate step coverage for a variety of geometries and deposition conditions. In principle, no adjustable parameters in these simulations are necessary and surprisingly good agreement between experiments and calculations has been shown (see also below). Analytical solutions for general cases are not possible but if certain simplifications are accepted, ${ }^{6}$ the

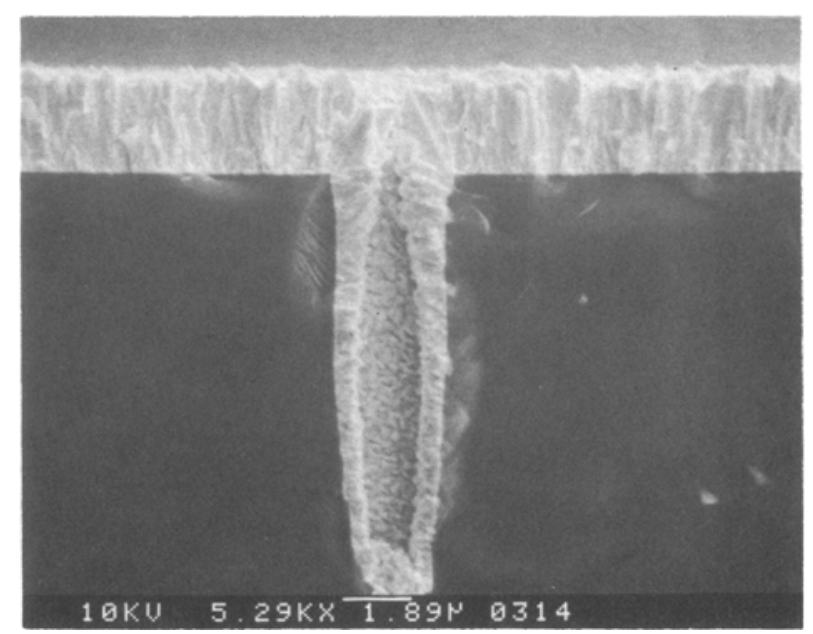

Fig. 1. SEM cross section of the tungsten film profile in a contact hole. In this case the conditions during deposition were chosen such that the step coverage is less than $100 \%$ and the keyhole (void) formation occurred. Such voids can cause the contact area to be destroyed during subsequent process steps such as blanket tungsten etch back or can cause reliability problems in the interconnect system. 
following equation for step covarage in a contact opening with initial radius $R_{0}$ and initial depth $L_{0}$ can be obtained ${ }^{7}$

$$
\text { Step coverage }=1-\left[L_{\mathrm{o}} / R_{\mathrm{o}}\right] A
$$

where $\mathrm{A}$ is not dependent on $L_{\mathrm{o}}$ or $R_{\mathrm{o}}$ but is a function of the deposition conditions (temperature, $\mathrm{H}_{2}$ and $\mathrm{WF}_{6}$ concentrations). A more accurate treatment ${ }^{7}$ leads to a more complicated expression, which gives, however, the same trends for the step coverage in terms of $L_{\circ}$ and $R_{0}$. Thus, Eq. 2 suggests that smaller contacts (smaller $R_{\mathrm{o}}$ ) are more difficult to fill than larger contacts of the same depth (equal $L_{0}$ ) since the calculated step coverage will be smaller for smaller diameter contact. At this time it is important to realize that step coverage defined in this way will not necessarily describe the size of the void (see Fig. $2 b$ for a definition) which will be present in the contact if the step coverage is less than $100 \%$. Below it will be shown that under certain conditions the size of the void will not depend on the radius of the contact (at constant depth), however, the step coverage does (because $L_{0} / R_{\mathrm{o}}$ varies with radius at constant $L_{\mathrm{o}}$ ).

In the discussion below, the Thiele modules will be useful as shown by MeConica and Churchill ${ }^{8}$

$$
h_{\mathrm{T}}=\sqrt{[}\left[k^{\prime \prime} L_{\mathrm{t}}^{2} /\left(C_{\mathrm{WF} 6} D_{\mathrm{WF} 6} R_{\mathrm{t}}\right)\right]
$$

where $k$ " is the hetergeneous rate constant, $L_{t}$ is the contact depth at time $t, R_{\mathrm{t}}$ the contact radius at time $t, C_{\mathrm{WF} 6}$ the concentration of $\mathrm{WF}_{6}$ at the opening of the contact and $D_{\mathrm{WF} 6}$ the Knudsen diffusion coefficient of $\mathrm{WF}_{6}$. It can be shown that for a pore the concentration of $\mathrm{WF}_{6}$ at depth $L_{t}$ goes to zero when $h_{\mathrm{T}}=\sqrt{ } 2$. When this condition is reached the deposition rate at the contact base will drop to zero and the step coverage of the remaining portion of the deposition will be smaller than $100 \%$, i.e. void formation will occur. Note that the only time dependent variables in Eq. 3 are $L_{\mathrm{t}}$ and $R_{\mathrm{t}}$.

\section{Computational and Experimental}

For the simulation of the tungsten growth in the contact holes a numerical method is applied. Basically, a mass balance between the diffusion of reactants and the consumption of reactants at the surface is established. It has been confirmed in several independent studies, that excellent agreement between experimental and calculated step coverage can be obtained using these simple considerations. ${ }^{2,3.7-9}$ The details of the calculations used in this work have been described elsewhere. ${ }^{9}$ In that study no correction was made for the inherent curvature of the tungsten growth at the mouth of the contact. In the current study we wanted to study the effect of the curvature and therefore, the changing profile at the mouth of the contact during growth has been incorporated in the calculation. This is accomplished by calculating the curvature of the tungsten growth at the edge of the contact opening. The growth from the corner is the same in all directions defining the curvature.

The modeling results were obtained on a grid of 80 points uniformly distributed over the entire length of the contact. The time steps were chosen on the basis that a reactant concentration did not change more than $0.05 \%$ from the

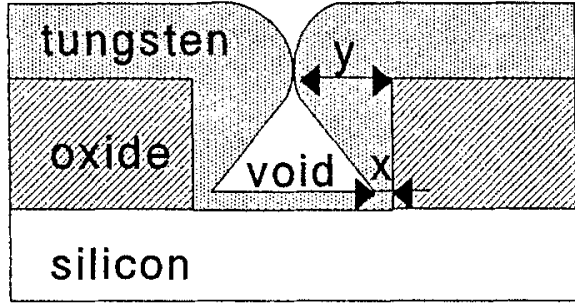

Step coverage $=(x / y)^{*} 100 \%$

(a)

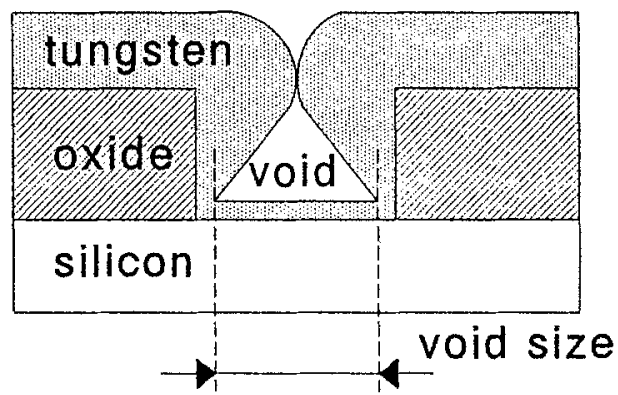

(b)

Fig. 2. Definition of step coverage (a) and of void size (b). This definition is arbitrary, other definitions in use in the literature might work as well.

previous calculation cycle. The maximum time step was set to $1 \mathrm{~s}$.

The deposition experiments, as depicted in Fig. 6, were done in an experimental cold wall reactor and on substrates described in detail in Ref. (9). The deposition conditions were: $\mathrm{WF}_{6}$ flow $=20 \mathrm{sccm}, \mathrm{H}_{2}$ flow $=500 \mathrm{sccm}$, Ar flow $=50 \mathrm{sccm}$, total pressure $=133 \mathrm{~Pa}$ and wafer temperature $=$ $703 \mathrm{~K}$. These conditions lead to a tungsten deposition rate of $87.5 \mathrm{~nm} / \mathrm{min}$. In the actual simulations the wafer temperature had to be adjusted from $703 \mathrm{~K}$ to $698 \mathrm{~K}$ in order to get agreement between experimental and calculated deposition rates. This small discrepancy is not unreasonable given the fact that precise wafer temperature determination in a cold wall reactor suffers from an inherent inaccuracy. ${ }^{10}$

\section{Results and Discussion}

Step coverage and contact size (surface curvature not included).-In Fig. 3 we show a schematic representation (as it is actually implicitly assumed in analytical calculations) of the film thickness profile at several times $t_{1}$, $t_{2}$, etc. during deposition. In this case we start with the deposition conditions such that the step coverage will be $100 \%\left(h_{\mathrm{T}}<\right.$ $\sqrt{2})$. Note that during the filling of the contacts the depth $\left(L_{t}\right)$ of the remaining aspect ratio will be identical to the

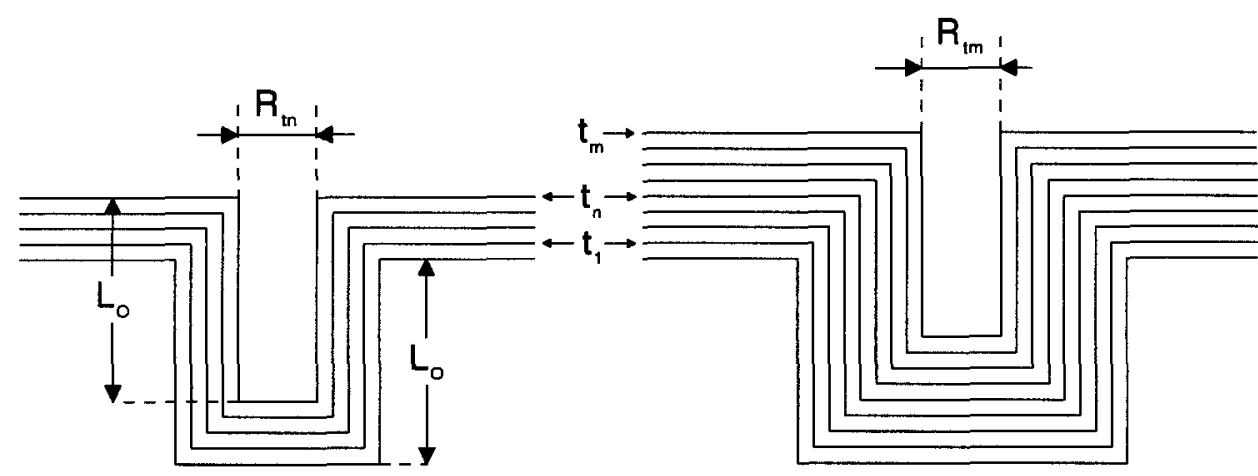

(a) (b)
Fig. 3. Schematic sketch of hole fill process for two contacts of the same depth but of different radius at several stages $t_{1}, t_{2}$ etc. If the deposition conditions are such that $h_{\mathrm{T}}<\sqrt{2}$ for the periods $0<t<t_{\mathrm{n}}$ and $0<t<t_{\mathrm{m}}$ and $t_{\mathrm{n}}$ and $t_{\mathrm{m}}$ are chosen such that $R_{\mathrm{tm}}=R_{\mathrm{tm}}$ the aspect ratio of the remaining pore will be equal: $L_{0} / \boldsymbol{R}_{\mathrm{m}}=L_{0} / \boldsymbol{R}_{\mathrm{tm}}$. If during the remaining portion of the deposition the condition $h_{\mathrm{T}}=$ $\sqrt{ } 2$ occurs, it is clear that the resulting void in Fig. $3 a$ and $b$ would be the same. 


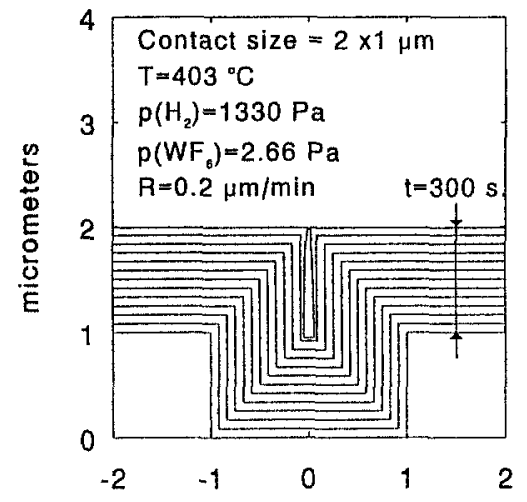

micrometers

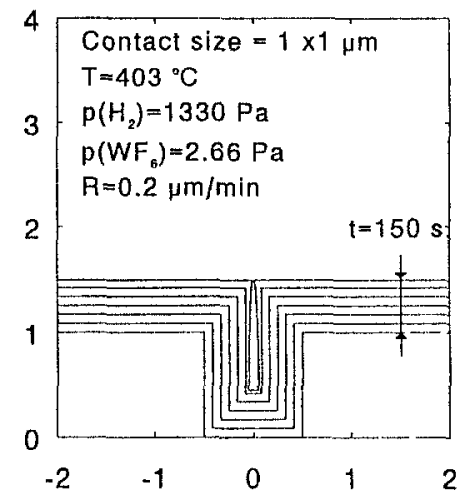

micrometers

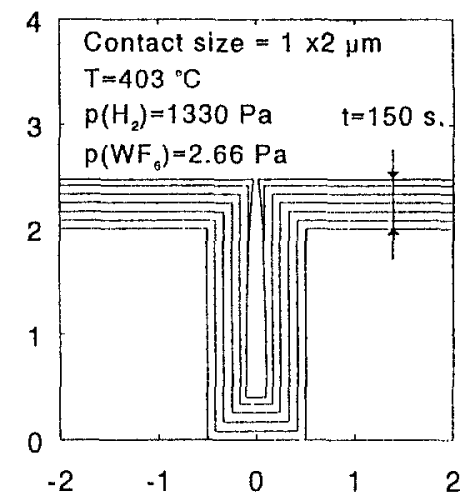

micrometers

\begin{tabular}{llll}
\hline Aspect ratio & 0.5 & 1.0 & 2.0 \\
Void size & $0.11 \mathrm{um}$ & $0.11 \mathrm{um}$ & $0.2 \mathrm{um}$ \\
Step cov. & $94 \%$ & $91 \%$ & $79 \%$ \\
\hline
\end{tabular}

Fig. 4. Actual simulation of the hole filling process of three contacts with different aspect ratios. No surface curvature of the growing film is included. The depth of the first two contacts is the same, whereas the radius is the same for the last two contacts. The conditions are chosen such that void formation will occur. The void size does not depend on the contact radius but it does depend on the contact depth.

contact's original depth $\left(L_{0}\right)$ but that the radius $R_{\mathrm{t}}$ diminishes because of the growth at the side walls. The result is that the aspect ratio $L_{\mathrm{t}} / R_{\mathrm{t}}$ will increase during growth. Therefore, $h_{\mathrm{T}}$ starts to approach the value $\sqrt{ } 2$ and the deposition rate in the bottom of the contact starts to drop: the formation of a void ("keyhole") starts. Rather trivial, but not generally recognized, is the fact that the limiting aspect ratio is independent of the radius of the contact but only depends on $L_{0}$. In other words the size of the keyhole depends only on the depth of the via and not on its aspect ratio. Therefore, the fundamental limitation of the fill capability of the blanket tungsten/etch back technique is not the initial aspect ratio of the contact but merely its depth. Thus in VLSI designs, one should carefully watch the tendency to make the dielectric films thicker. In Fig. 4 we have actually simulated the hole filling process for three different aspect ratios: $0.5,1$, and 2 . The conditions are chosen such that the step coverage is marginal for the $1 \mu \mathrm{m}$ deep contact. It is nicely illustrated here that the size of the keyhole is independent of the radius of the contact (compare $4 \mathrm{a}$ and $b$ ) but depends upon the depth (compare $4 a$ and $b$ ) of the contact. The step coverage as defined in Fig. 2 shows a different trend and follows closely the initial aspect ratio of the contact. We believe that the real concerns (i.e. etch back complications and reliability problems) caused by a deposition with step coverage less than $100 \%$ is more adequately described by the size of the keyhole rather than the value of the step coverage.

The influence of surface curvature on void formation.The discussion above is correct for the case that the radius $R_{\mathrm{t}}$ is contant over the entire depth $L_{0}$ (or $L_{\mathrm{t}}$ if the step coverage is less than $100 \%$ ). For real cases this is not an accurate description. In Fig. 5 we have depicted the result of simulations of the actual thickness profiles during several stages of the growth process for a small and large diam contact with identical depths ( $5 \mathrm{a}$ and $\mathrm{b}$ ) while incorporating the effect of surface curvature. It is clear that $R_{t}$ becomes now a non-trivial function of the depth. This implies that the radius $R_{\mathrm{t}}$ at which the condition $h_{\mathrm{T}}=\sqrt{2}$ is reached (i.e. at

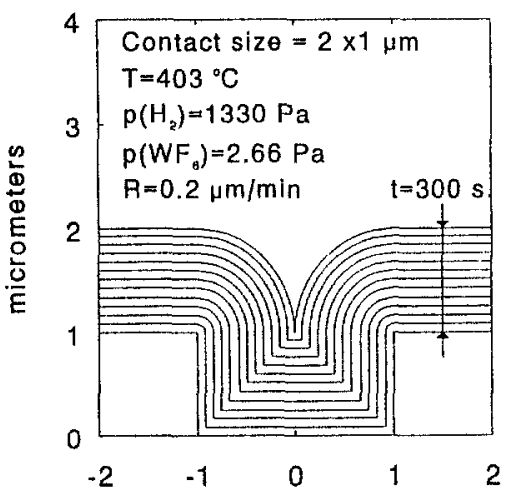

micrometers

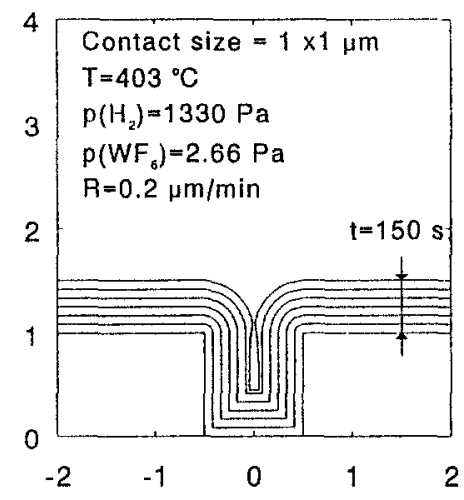

micrometers

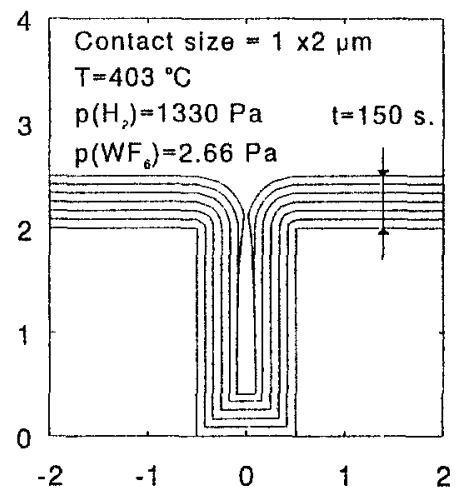

micrometers

\begin{tabular}{llll}
\hline Aspect ratio & 0.5 & 1.0 & 2.0 \\
Void size & $0.0 \mathrm{um}$ & $0.1 \mathrm{um}$ & $0.2 \mathrm{um}$ \\
Step cov. & $100 \%$ & $91 \%$ & $81 \%$ \\
\hline
\end{tabular}

Fig. 5. Simulation of the hole filling process incorporating surface curvature. The same holes as in Fig. 4. are shown. Again the conditions are such that void formation will occur. It can be clearly seen that the surface curvature has an effect on the void size but only at the smaller aspect ratios. 

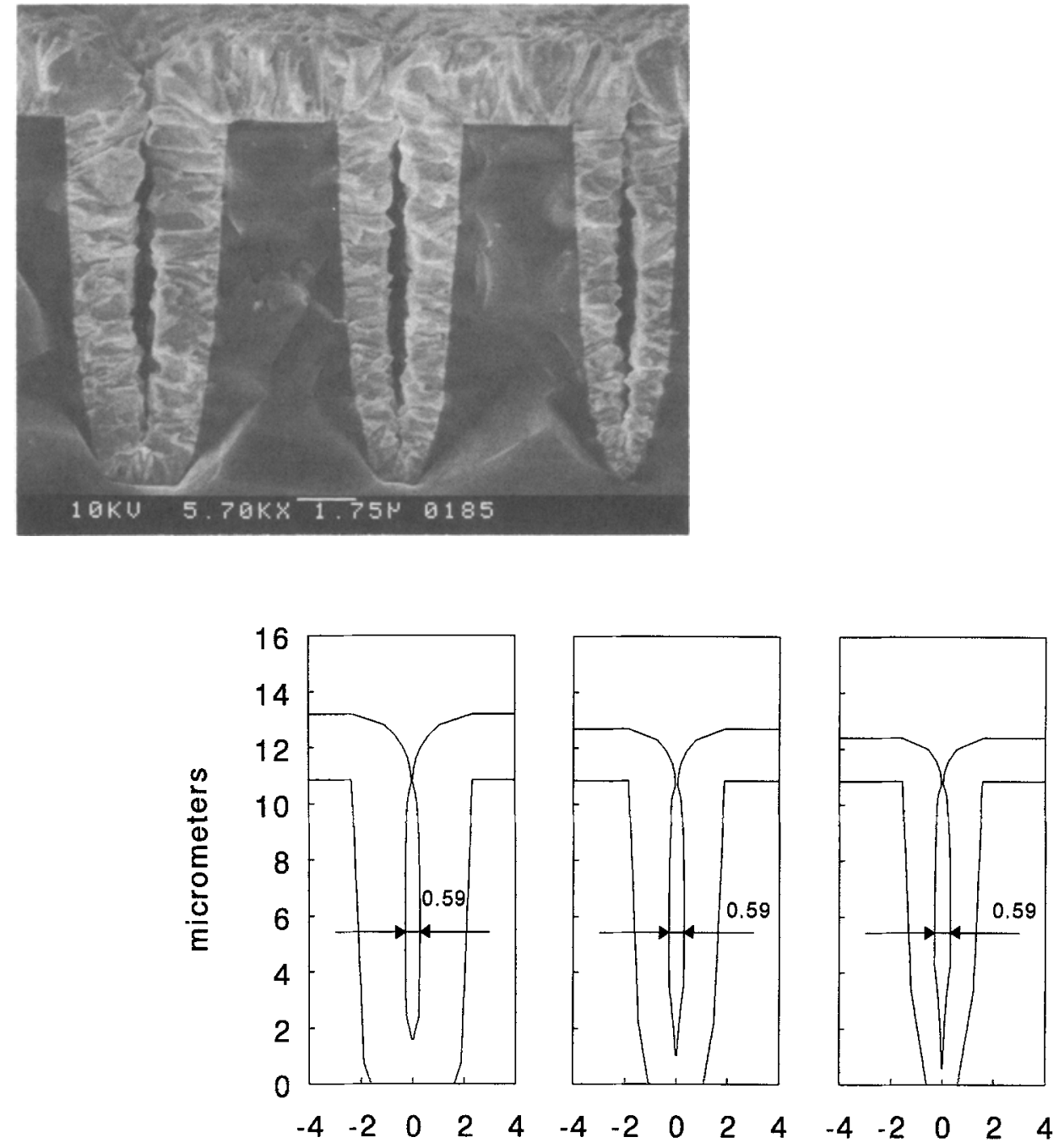

micrometers

micrometers
Fig. 6. CVD-tungsten film profiles in trenches of the same depth but with various widths (a) and the simulation of these conditions (b). Note that the width of the void there measured at $6 \mu \mathrm{m}$ depth because the trench profile is distorted near the bottom) does not depend on the width of the trench. Trench dimensions: $4.73 \times 10.85 \mu \mathrm{m}$ (left) $3.77 \times$ $10.85 \mu \mathrm{m}$ (middle), and $3.15 \times$ $10.85 \mu \mathrm{m}$ (right). The dimension of the voids is in micrometers. See text for more details.

\section{void simulation $\quad 0.59$ \\ 0.59

the onset of void formation) is smaller for the larger (i.e. larger radius) contact. This agrees with the practical experience that smaller contacts tend to have a higher risk for void formation than do larger contacts of identical depth. Note that in the case where curvature is not taken into consideration (Fig. $4 \mathrm{a}$ and $\mathrm{b}$ ) the size of the void does not depend on contact radius for contacts of the same depth. In Fig. $5 \mathrm{c}$ we have simulated the thickness profiles during several stages of the deposition process but now for a contact with an aspect ratio of 2 and the same radius as the contact in Fig. 5 b. Clearly the void size becomes worse with the deeper contact.

Further, it is interesting to note that the influence of the curvature becomes less with deeper contacts. Compare Fig. $4 \mathrm{c}$ and Fig. 5c. The width of the void at the bottom in both cases is nearly identical. Therefore, for deep, high aspect ratio ( $>2$ ) contacts the simplified description (no curvature) is adequate as also was applied in Ref. 9.

\section{Experimental Verification}

In the discussion above, we have already pointed out that the followed procedures to simulate deposition profiles have been verified several times in recent literature and found to give good agreement between simulations and actual experimental cases. Therefore, we will verify the re- sults here only for a limited amount of cases. In the experiments we have used trenches rather than holes (see Fig. 6) for two reasons: 1) It is extremely difficult to get accurate film thickness profiles in the contact from SEM cross sections. Slightly misaligned cleaves (not through the center or cleaves not parallel to axis of symmetry) will give strongly distorted film thickness profiles in the contact pore. In contrast, this is not true for trenches where proper (easily) alignment of the sample in the SEM will automatically correct for any not ideal cleave orientation: and 2) It has been found recently that there exists a very strong correlation between the step coverage in the contact holes and trenches (if both have the same aspect ratio.) ${ }^{9}$ Using this correlation results obtained in trenches will be identical to those in contacts with only a given lower value of the step coverage.

As argued above it can be seen from the simulated profiles that the size of the void is indeed independent of the trench width (i.e. contact diam). The accuracy of the experimental cross sections is somewhat hampered $( \pm 0.05 \mu \mathrm{m})$ because of the roughness of the tungsten film but shows nevertheless the same trend with trench width as do the simulations. Within experimental error the size of the void in the experiment is in agreement with the value obtained from the simulations. 


\section{Conclusions}

In this paper we have studied in detail the mechanism of the CVD-W contact fill process and the usefulness of the concept of step coverage. This has been done by simulating the process of contact fill with and without the inclusion of surface curvature. Based on the outcome of the simulations and experiments we conclude that: the effect of surface curvature on the hole filling process is substantial for contacts with an aspect ratio $<1.0$. This curvature effect is not taken into account in analytical solutions for the step coverage. For contacts with an aspect ratio $>2.0$ the effect of the curvature on void formation becomes negligible; for contacts with an aspect ratio $>2.0$ the depth is the only parameter which determines the size of the keyhole in the case of the step coverage being less than $100 \%$. Therefore, the real limitation for the blanket CVD-W process to fill contacts is contact hole depth rather than contact diameter. The value of the step coverage does not necessarily indicate the size of the void. However, void size is the true parameter which describes the associated risks emerging in subsequent process steps such as blanket tungsten etch back. Therefore, the size of the void (only dependent on process conditions and contact depth but not on contact diam) is a better parameter to describe the conformality of the deposition rather than the value of the step coverage (which is very dependent on contact diameter, contact depth, and process conditions).

Although this study has been performed using CVD-W, the presented principles are quite general and do certainly apply to other chemical vapor depositions such as silicon oxide, silicon nitride, and tungsten silicide. In each of these cases, however, the reaction kinetics and the effect of surface diffusion have to be understood before an adequate description of the fill process can be given.

\section{Acknowledgment}

The authors wish to thank Norm Zetterquist and Martin Henry for reading the manuscript and useful suggestions.

Manuscript submitted Aug. 31, 1992; revised manuscript received March 4, 1993

Genus, Inc. assisted in meeting the publication costs of this article.

\section{REFERENCES}

1. See for example J. E. J. Schmitz in Chemical Vapor Deposition of Tungsten and Tungsten Silicides for VLSI/ULSI Applications, Noyes Publications, Park Ridge, NJ (1991), and references therin.

2. A. Hasper, J. Holleman, J. Middelhoek, and C. R. Kleijn, in Tungsten and Other Advanced Metals for VLSI/ULSI Applications, S. S. Wong and S. Furukawa, Editors, p. 127, MRS, Pittsburgh, PA (1990).

3. J. E. J. Schmitz, W. L. N. van der Sluys, and A. H. Montree, ibid, p. 117 (1990).

4. E. K. Broadbent and C. L. Ramiller, This Journal, 131, 1427 (1984).

5. C. M. McConica and K. Krishnamani, ibid., 133, 2542 (1986).

6. See Ref. 1, p.30.

7. S. Chatterjee and C. M. McConica, This Journal, 137, 328 (1990).

8. C. M. McConica and S. Churchill, in Tungsten and Other Refractory Metals for VLSI Applications, E. K. Broadbent, Editor, p. 257, MRS, Pittsburgh, PA (1988).

9. A. Hasper, J. Holleman, J. Middelhoek, C. R. Kleijn, and C. J. Hoogendoorn, This Journal, 138, 1728 (1991).

10. A. Hasper, J. E. J. Schmitz, J. Holleman, and J. F. Verwey, J. Vac. Sci. Technol. A, 10, 3193 (1992).

\title{
A Highly Selective, Chlorofluorocarbon-Free GaAs on AlGaAs Etch
}

\author{
L. E. Smith* \\ AT\&T Bell Laboratories, Solid State Technology Center, Breinigsville, Pennsylvania 18031
}

\section{ABSTRACT}

A highly selective reactive ion etching process using $\mathrm{SiCl}_{4}, \mathrm{CF}_{4}, \mathrm{O}_{2}$, and $\mathrm{He}$ is reported. The selectivity of the etch, which is adjustable, ranges from 308:1 to $428: 1$ for $\mathrm{GaAs}$ to $\mathrm{Al}_{011} \mathrm{Ga}_{0.89} \mathrm{As}$. This variability in selectivity is achieved by adjusting the helium flow rate. One very attractive feature of this etch is that it uses no chlorofluorocarbons and therefore complies with future bans on these substances imposed at both federal and corporate levels. ${ }^{1-3}$ The etch is demonstrated on a GaAs field effect transistor structure with an underlying $\mathrm{Al}_{0.11} \mathrm{Ga}_{0.89}$ As stop-etch layer. The etch can be used for both anisotropic and isotropic applications.

Dichlorodifluoromethane $\left(\mathrm{CCl}_{2} \mathrm{~F}_{2}\right.$, aka Freon-12®) is probably the most widely used etchant in use today for selectively etching GaAs over AlGaAs. ${ }^{4-8}$ Freon-12 is nontoxic, nonflammable, noncorrosive, and consequently a very suitable species for the etching of GaAs and the above mentioned selective etching. However, Freon-12 is a class 1 $\mathrm{CFC}$ with a high ozone depleting potential (ODP) and with recent activities geared to revise the Clean Air Act, will probably not be manufactured after $1995 .^{3}$ As a result, processors are finding alternatives for etching. There are scores of chlorine and fluorine-based species from which to choose. Hydrochlorofluorocarbons (HCFCs) are one such alternative, but these too are classified as ozone depleting chemicals (ODCs), albeit they have much lower ODPs, and are subject to manufacturing restrictions and cessation by $2015,{ }^{9}$ with impending restrictions which will probably push up that date to $2005 .{ }^{3}$ Other alternatives rely on mix-

\footnotetext{
* Electrochemical Society Active Member.
}

ing chlorine-based and fluorine-based species or chlorinebased and oxygen-based species to achieve selectivity, a practice used even before the beginning of the CFC ban. ${ }^{10}$ One very promising combination of these species is reported herein. This combination of $\mathrm{SiCl}_{4}, \mathrm{CF}_{4}, \mathrm{O}_{2}$ and $\mathrm{He}$, when used under the right conditions produces an etch which can be easily tailored to many needs to terms of isotropy and selectivity.

\section{Experimental}

The etching was done in a parallel plate, turbo-pumped system with an aluminum chamber [Oxford-Plasma Technology, micro-p reactive ion etcher]. The chamber was modified from the original configuration: from a 7 in. bottom electrode with $21 / 2$ in. ports, valves, and vacuum lines to a $9 \mathrm{in}$. bottom electrode with 4 in. ports, valves, and vacuum lines. This modification increased the gas throughput capability by a factor of five allowing high flow, low 